\title{
Comparisons of foramen ovale flap in the fetuses with true and false positive diagnosis of coarctation of the aorta
}

\author{
Ruan Peng"^ ${ }^{\#}$ Qiao Zheng", Miao He, Jian-Hong Shang, Hong-Ning Xie \\ Department of Ultrasonic Medicine, Fetal Medical Centre, The First Affiliated Hospital of Sun Yat-sen University, Guangzhou, China
}

Contributions: (I) Conception and design: HN Xie; (II) Administrative support: JH Shang; (III) Provision of study materials or patients: Q Zheng; (IV) Collection and assembly of data: R Peng, M He; (V) Data analysis and interpretation: R Peng; (VI) Manuscript writing: All authors; (VII) Final approval of manuscript: All authors.

\#These authors contributed equally to this work.

Correspondence to: Hong-Ning Xie, PhD. Department of Ultrasonic Medicine, Fetal Medical Centre, The First Affiliated Hospital of Sun Yat-sen University, Zhongshan 2nd Road 58\#, Guangzhou, China. Email: xiehn@mail.sysu.edu.cn.

Background: Accurate diagnosis of coaractation of aorta $(\mathrm{CoA})$ remains challenging because of its relatively low sensitivity and specificity. It is difficult to distinguish true CoA from a normal physiological right-sided dominance or ventricular discrepancy caused by intracardiac minor anomalies. Redundant foramen ovale flap (RFOF) may cause ventricular disproportion.

Methods: All fetuses suspected with CoA on routine screening ultrasound were retrospectively reviewed and allotted into two groups: postnatally confirmed CoA (true positive group) and prenatally suspected CoA but without CoA postnatally (false positive group). Sixty-nine normal fetuses were included as a normal group (normal group). The diameters of FOF and left atrium (LA) were measured in the four-chamber view and FOF/LA ratio $\geq 0.65$ was considered as RFOF. Cardiac parameters between groups were compared.

Results: Fifty-seven fetuses undergoing echocardiography for suspicion of CoA were enrolled; 11 (19.2\%) had CoA postnatally. A significant linear relationship was identified between ventricular discrepancy degree and FOF prominence $(\mathrm{P}<0.001, \mathrm{R}=0.48)$. A significant linear relationship was also identified between the RFOF and disproportion of the great arteries $(\mathrm{P}<0.001, \mathrm{R}=0.42)$. FOF prominence significantly differed between groups true positive and false positive $(\mathrm{P}<0.001)$. RFOF occurred significantly differently in the true positive and false positive groups $(56.5 \%$ vs. $0.91 \%, \mathrm{P}=0.002)$. Cardiac parameters, including the aortic valve (AO) z-score $(\mathrm{P}=0.785)$, aortic isthmus (AOi) z-score $(\mathrm{P}=0.944)$, pulmonary artery $(\mathrm{PA}) \mathrm{z}$-score $(\mathrm{P}=0.693)$, $\mathrm{PA} / \mathrm{AO}$ ratios $(\mathrm{P}=0.055)$, left ventricle $(\mathrm{LV}) \mathrm{z}$-score $(\mathrm{P}=0.192)$ and right ventricle $(\mathrm{RV}) / \mathrm{LV}$ ratios $(\mathrm{P}=0.225)$, were comparable between fetuses with and without CoA after birth except RV z-score (P=0.035).

Conclusions: There is no statistical difference of cardiac parameters between fetuses with and without CoA after birth except RV diameter. The proportion of fetuses with RFOF is significantly greater in patients without CoA. Fetal echocardiography including bulging of the FOF in the LA should be investigated for suspected CoA to decrease false positive diagnosis of CoA.

Keywords: Redundant foramen ovale flap (RFOF); coarctation of the aorta (CoA); prenatal diagnosis; false positive diagnosis

Submitted Jun 16, 2021. Accepted for publication Dec 29, 2021; Published online: 17 Jan 2022.

doi: 10.21037/qims-21-644

View this article at: https://dx.doi.org/10.21037/qims-21-644

\footnotetext{
^ ORCID: 0000-0002-8548-9663.
} 


\section{Introduction}

The foramen ovale is an oval opening in the middle third of the inter-atrial septum that allows communication from the right atrium (RA) to the left atrium (LA); thus, oxygenenriched blood can be shunted to the left ventricle (LV) and then into the upper part of the body, including the cerebral and coronary arteries. The foramen ovale is covered by a free flap from the septum primum, which bulges into the LA. If the foramen ovale flap (FOF) balloons at least halfway across the LA, it will be considered as a redundant FOF (RFOF) (1). The reported frequency of RFOF in the referral cohort for echocardiography was $0.2-1.0 \%(2-4)$; in fetuses with restrictive foramen ovale, the reported frequency was about $30 \%$ (5).

A recently published study showed that the presence of RFOF may simulate coarctation of the aorta (CoA) (6). CoA is one of the most common congenital heart diseases in neonates and children, and may require immediate surgery after birth. Previous studies reported that prenatal diagnosis of CoA lacked sensitivity and specificity, and less than one-third of cases were detected via prenatal ultrasound screening $(7,8)$. False positive diagnosis of CoA can cause parental anxiety and may change delivery plans for prenatal care. Vena et al. (6) demonstrated that RFOF determined ventricular asymmetry and reduced the diameter of the aortic isthmus (AOi). However, the difference in FOF prominence between fetuses with false positive and true positive CoA was not definite.

Thus, the primary aim of this study was to assess the relationship between RFOF prominence and discrepancy between cardiac and physical functions, including that of the ventricles and great arteries. The secondary aim was to compare the difference in distribution of FOF in cases with a true positive diagnosis versus those with a false positive diagnosis of CoA.

We present the following article in accordance with the STROBE reporting checklist (available at https://qims. amegroups.com/article/view/10.21037/qims-21-644/rc).

\section{Methods}

\section{Study design and participants}

We retrospectively reviewed all cases initially suspected to have CoA at routine screening ultrasound between January 2014 and July 2020 in our tertiary referral center. Written informed consent was obtained from their legal representatives (one person of their parents) because our participants were neonates or toddlers and the study was approved by the ethics committee of our institution. The study was conducted in accordance with the Declaration of Helsinki (as revised in 2013).

Fetal echocardiography and detailed anatomy evaluation were performed in each case with a suspicion of CoA. Exclusion criteria were fetuses with chromosomal abnormalities; coexistent intracardiac anomalies, including persistent left superior vena cava and ventricular septal defect; fetuses with growth impairment; cases lost to follow up; terminated pregnancy or selective reduction; and cases with insufficiently clear ultrasound images. However, cases with ventricular septal defect that could not be visualized with gray scale images were recruited. Postnatal echocardiography and/or computed tomography (CT) findings and clinical records were available for all patients. Fetal magnetic resonance imaging (MRI) or cardiac MRI was not performed afterwards. All fetuses with suspected CoA were allotted into two groups based on the postnatal results: cases with postnatally confirmed CoA (true positive group) and cases with prenatally suspected CoA but without CoA after birth (false positive group). Additionally, 69 normal fetuses were recruited as the normal group (normal group).

\section{Echocardiography and ultrasound measurements}

Fetal echocardiography was performed using a GE Voluson 730, E8, or E10 (GE Healthcare, Zipf, Austria) ultrasound machine equipped with a 4-8-D RAB probe (GE Healthcare). Video clips or three-dimensional volume datasets of fetal cardiac tissue were reviewed to perform the measurements. The 4D viewer software (version 17; GE Healthcare) was used for the measurements. A single frame that clearly showed the prominence of the FOF was chosen for investigation and measurements. Both investigators (R.P. and Q.ZH.) who performed the ultrasound examinations were blinded to the clinical results. For repeatability analysis, 30 cases were randomly selected from the control group (normal group). Ultrasound measurements were carried out in these 30 cases by a first operator (R.P.). All images were then processed by both a second operator (Q.ZH.) and the first operator to assess the interobserver and intraobserver repeatability.

The following cardiac parameters were assessed in this study: transverse diameter of the LA, RA, LV, and right ventricle (RV); aortic valve (AO); pulmonary artery $(\mathrm{PA})$; and AOi. We measured the maximum transverse 


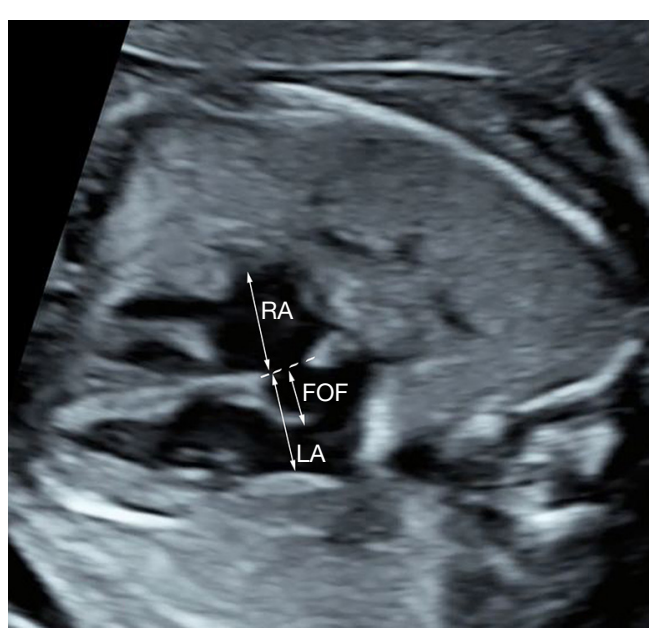

Figure 1 Four-chamber cardiac view in a fetus of 29 gestational weeks. The image shows the methodology for the measurement of the FOF and LA. FOF/LA ratio was obtained by dividing the maximum diameter of the FOF with the transverse diameter of the LA in the four-chamber view. FOF, foramen ovale flap; LA, left atrium; RA, right atrium.

diameters of the LA and RA in the four-chamber view just above the atrioventricular valve orifice at the end of the systolic phase of the cardiac cycle. Measurements of the maximum transverse diameters of the $\mathrm{LV}$ and $\mathrm{RV}$ were made in the four-chamber view just below and parallel to the atrioventricular valve orifice in the end-diastolic phase. The diameters of the AO and PA (inner edge to inner edge) were taken from the outflow tract views of the left and RVs at the end of the systolic phase. The maximum diameter of the FOF was measured according to the approach proposed by Vena et al. (6). A line was drawn along the interatrial septum, and then the maximum diameter of the FOF was measured from the outer edge of the more prominent part of the FOF to this line (Figure 1). AOi diameter was measured in the three vessel and trachea view. All z-scores were computed for the fetal femur length as described previously (9). In addition, the relative size of the transverse diameters of the $\mathrm{RV}$ and $\mathrm{LV}$ ( $\mathrm{RV} / \mathrm{LV}$ ratio), the relative size of the $\mathrm{PA}$ and $\mathrm{AO}(\mathrm{PA} / \mathrm{AO}$ ratio), and the transverse diameter of the FOF and LA (FOF/LA ratio) were also obtained. The flow direction across the AOi was also recorded, such as forward, mixed, or reversed.

\section{Statistical analysis}

Statistical analysis was performed using SPSS version 25.0
(IBM Corp., Armonk, NY, USA). The agreement between the two observers was depicted using Bland-Altman plots, and the interclass correlation coefficient (ICC) was calculated to evaluate the inter- and intraobserver reliability. For clinical application, ICC repeatability was considered as slight $(0-0.2)$, fair $(0.21-0.4)$, moderate $(0.41-0.6)$, substantial (0.61-0.8), and almost perfect (0.81-1.0) (10). The relationship between $\mathrm{RV} / \mathrm{LV}$ ratio and FOF prominence, $\mathrm{PA} / \mathrm{AO}$ ratio and FOF prominence were both analyzed by using Pearson correlation test. Comparisons of categorical variables are reported as percentages and analyzed using the chi-square test or Fisher's exact test. The median and range were determined and the Mann-Whitney $\mathrm{U}$ test was used for analysis of non-normally distributed variables between different subgroups. The cases that were lost to follow up were excluded for the final analysis. Statistical significance was set at $\mathrm{P}<0.05$. The study size was acquired according to the formula that $\mathrm{n}=\left[\left(\mathrm{Z}_{1-\alpha / 2} \times \sigma\right) / \delta\right]^{2}$, $\alpha=0.05, \delta=0.3, \sigma=1.0$.

\section{Results}

Of the 104 fetuses suspected to have CoA prenatally, 47 cases were excluded due to termination of pregnancy or selective reduction $(n=37)$, loss to follow-up $(n=9)$, and intrauterine death $(\mathrm{n}=1)$. Finally, 57 fetuses undergoing echocardiography for the suspicion of CoA were enrolled; $11(19.2 \%)$ were confirmed to have CoA postnatally. Ten neonates required surgery, and one patient underwent expectant management until writing. The median maternal age was 32 [22-41], 33 [21-40] and 32 [22-39] years old in groups true positive (with CoA after birth), false positive (without CoA after birth), and normal, respectively $(\mathrm{P}=0.556)$. The median gestational age at examination was $28^{+6}\left(21^{+1}-36\right), 32^{+1}\left(23^{+1}-38^{+1}\right)$, and $29^{+4}\left(21^{+1}-37^{+3}\right)$ weeks in these three groups $(\mathrm{P}=0.124)$. Overall, five fetuses had small ventricular septal defects that were identified using color Doppler imaging. In 26 patients, forward flow at the aortic arch was identified. Mixed flow and reversed flow at the aortic arch were recorded in eight and 23 cases, respectively.

The agreement between different observers and intraand interobserver reliability for the measurement of fetal cardiac parameters, including LA, RA, LV, RV, PA, AO, AOi, and FOF are shown in Table 1. A relatively good reliability was found for all cardiac parameters except RA.

At first, we analyzed the 115 fetuses without CoA after birth (groups true positive and false positive). A significant linear relationship was identified between the degree of 
Table 1 The intra- and interobserver agreement and reliability for measurement of fetal cardiac parameters

\begin{tabular}{|c|c|c|c|c|}
\hline Parameter & Mean difference $95 \% \mathrm{Cl}(\mathrm{mm})$ & $95 \%$ LOA (mm) & Intraobserver ICC (95\% Cl) & Interobserver ICC (95\% Cl) \\
\hline Right atrium & $0.13(0.08$ to 0.19$)$ & -0.44 to 0.70 & $0.82(0.75$ to 0.89$)$ & $0.81(0.74$ to 0.88$)$ \\
\hline Left ventricle & $0.04(-0.01$ to 0.09$)$ & -0.26 to 0.29 & 0.94 (0.91 to 0.98$)$ & 0.93 (0.89 to 0.97$)$ \\
\hline Right ventricle & $0.05(-0.007$ to 0.1$)$ & -0.23 to 0.33 & 0.93 (0.87 to 0.98$)$ & 0.91 (0.85 to 0.95$)$ \\
\hline Aorta & $0.02(-0.02$ to 0.07$)$ & -0.28 to 0.24 & 0.94 (0.91 to 0.99$)$ & 0.92 (0.88 to 0.97$)$ \\
\hline Isthmus of aorta & $0.01(-0.04$ to 0.05$)$ & -0.23 to 0.25 & 0.90 (0.81 to 0.93$)$ & 0.89 (0.80 to 0.95$)$ \\
\hline Foramen ovale flap & $0.03(-0.03$ to 0.09$)$ & -0.31 to 0.38 & 0.89 (0.82 to 0.92$)$ & 0.87 (0.79 to 0.96$)$ \\
\hline
\end{tabular}

LOA, limits of agreement; ICC, intraclass correlation coefficient.
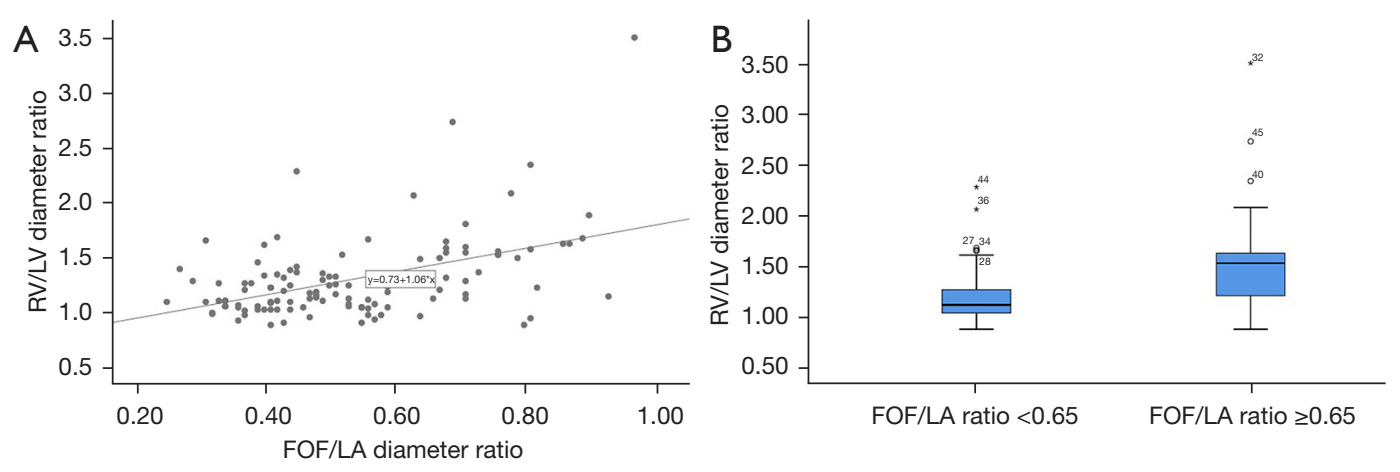

Figure 2 Relationship between the degree of ventricular discrepancy (RV/LV ratio) and FOF prominence in 115 fetuses without CoA after birth (normal group and false positive group). (A) Prominence of the FOF is expressed as a continuous variable with FOF/LA ratio or (B) as a categorical variable with $\mathrm{FOF} / \mathrm{LA}$ ratio $\geq 0.65 .{ }^{*},>\mathrm{Q} 3+1.5 \mathrm{IQR} ;{ }^{\circ},>\mathrm{Q} 3+3 \mathrm{IQR}$. RV, right ventricle; $\mathrm{LV}$, left ventricle; FOF, foramen ovale flap; CoA, coarctation of aorta; IQR, interquartile range.

ventricular discrepancy (RV/LV ratio) and FOF prominence, which was expressed as a continuous variable using FOF/ LA ratio $(\mathrm{P}<0.001, \mathrm{R}=0.48)$ or as a categorical variable using $\mathrm{FOF} / \mathrm{LA}$ ratio $\geq 0.65$ ( $\mathrm{P}=0.003)$, which was proposed by Vena et al. (6). Figure 2 demonstrates the relationship between ventricular discrepancy and FOF prominence. A significant linear relationship was also identified between the redundancy of the FOF and the disproportion of the great arteries, which was expressed as the $\mathrm{PA} / \mathrm{AO}$ ratio $(\mathrm{P}<0.001, \mathrm{R}=0.42$ and $\mathrm{P}=0.001$, respectively) (Figure 3).

And then, the fetuses with confirmed CoA (true positive group) were compared with fetuses with a false positive diagnosis of CoA (false positive group). The prominence of FOF, expressed as FOF/LA ratio, was significantly different between these two groups $(\mathrm{P}<0.001)$ (Figure 4). Video 1 shows a fetus with RFOF and suspected CoA with prenatal ultrasound screening at 29 gestational weeks.
However, the neonate was identified with normal aortic arch by using postnatal echocardiography (RFOF, false positive of CoA, prenatal ultrasound). RFOF was present in 26 patients $(56.5 \%)$ with a false positive diagnosis of CoA but only one patient $(0.91 \%)$ with a confirmed diagnosis of CoA $(\mathrm{P}=0.002)$. On the other hand, the cardiovascular parameters, including the $\mathrm{AO} z$-score ( $\mathrm{P}=0.785)$, AOi $\mathrm{z}$-score $(\mathrm{P}=0.944), \mathrm{PA} z$-score $(\mathrm{P}=0.693)$, $\mathrm{PA} / \mathrm{AO}$ ratios $(\mathrm{P}=0.055), \mathrm{LV}$ z-score $(\mathrm{P}=0.192)$ and $\mathrm{RV} / \mathrm{LV}$ ratios $(\mathrm{P}=0.225)$, were comparable in fetuses in groups true positive and false positive except $\mathrm{RV} z$-score $(\mathrm{P}=0.035)$.

Finally, we analyzed the 11 fetuses in true positive group and the 26 fetuses in false positive group with RFOF, defined as a FOF/LA ratio $\geq 0.65$. The degree of ventricular disproportion, expressed as the $\mathrm{RV} / \mathrm{LV}$ ratio, was similar between the 11 fetuses in true positive group and the 26 fetuses in false positive group with RFOF $(\mathrm{P}=0.065)$ 

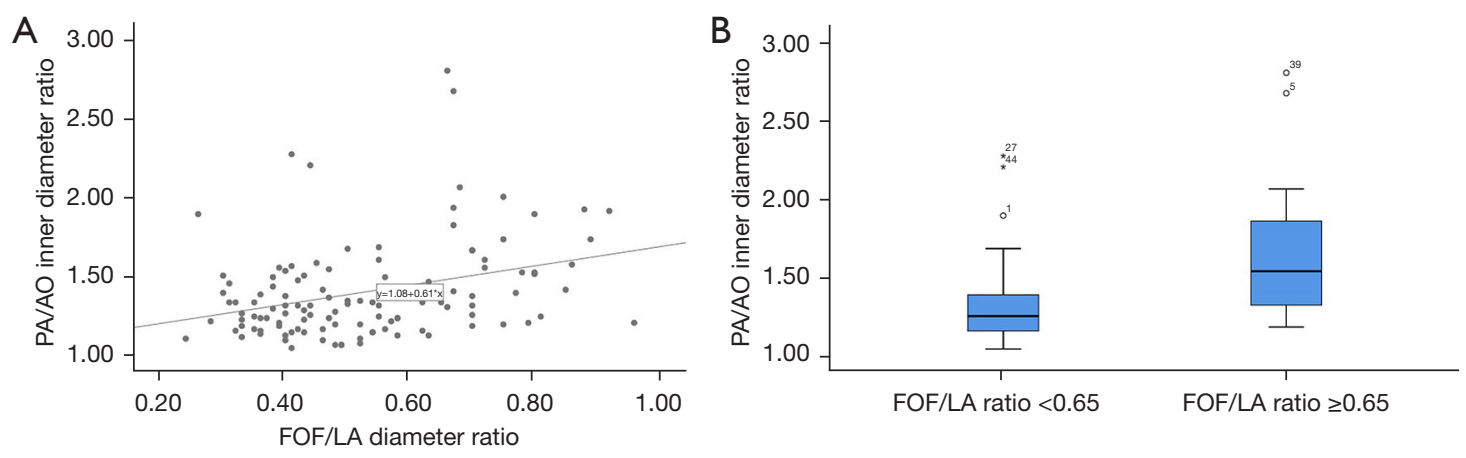

Figure 3 Relationship between the z-score of AOi and redundancy of the FOF in 115 fetuses without CoA after birth (normal group and false positive group). Redundancy of the FOF is expressed as (A) a continuous variable with FOF/LA ratio or (B) a categorical variable with

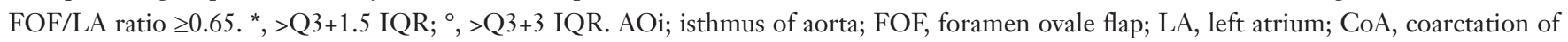
aorta; IQR, interquartile range.

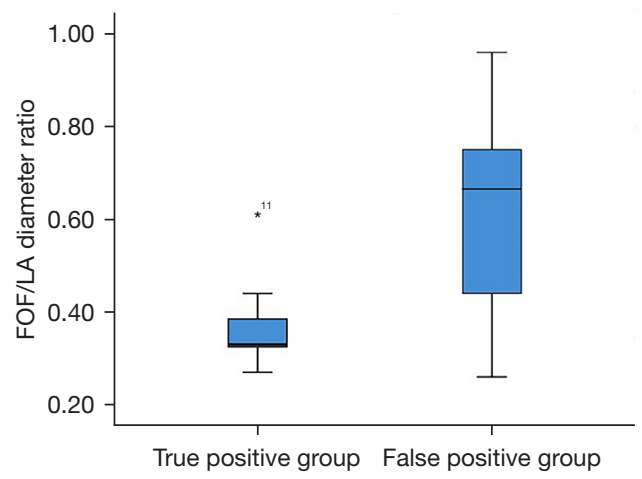

Figure 4 Box-and-whisker plot showing the distributions of FOF/ LA ratio in groups True positive and false positive. ${ }^{*},>\mathrm{Q} 3+1.5$ IQR. FOF, foramen ovale flap; LA, left atrium; true positive group, the fetuses without CoA after birth; false positive group, the fetuses with CoA after birth; IQR, interquartile range.

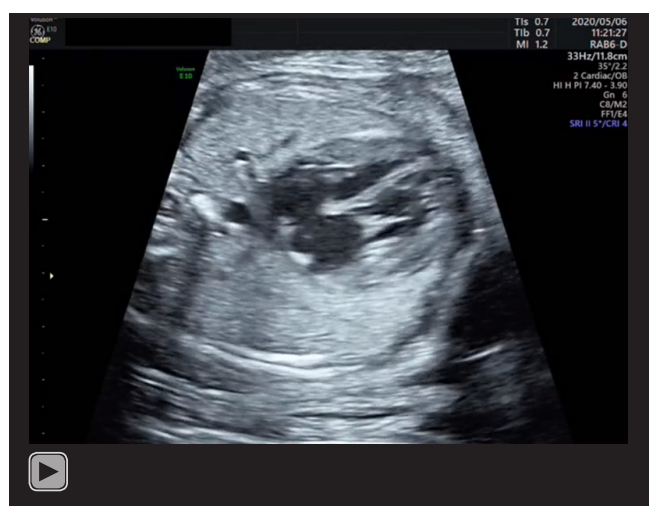

Video 1 A fetus with redundant foramen ovale flap and suspected coarctation of aorta with prenatal ultrasound screening at 29 gestational weeks.

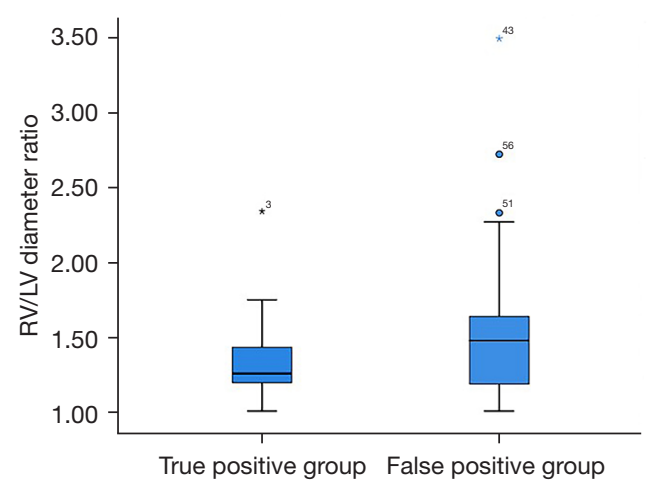

Figure 5 Box-and-whisker plot showing the distributions of the ratio of the right ventricle width to the left ventricle width (RV/ $\mathrm{LV}$ ratio) in groups true positive and false positive. *, $>\mathrm{Q} 3+1.5$ $\mathrm{IQR} ;{ }^{\circ},>\mathrm{Q} 3+3 \mathrm{IQR}$. RV, right ventricle; $\mathrm{LV}$, left ventricle; IQR, interquartile range.

(Figure 5). The AOi $(\mathrm{P}=0.987)$, the $\mathrm{PA}$ diameters, $(\mathrm{P}=0.907)$, the AO diameters $(\mathrm{P}=0.163)$, expressed as $\mathrm{z}$-scores, and the $\mathrm{PA} / \mathrm{AO}$ ratios $(\mathrm{P}=0.114)$ were also not significantly different between the 11 fetuses in true positive group and the 26 fetuses in false positive group with RFOF. The incidence of reversed flow in the aortic arch was not significantly different between fetuses with RFOF and those with confirmed CoA (38.4\% vs. 27.3\%, $\mathrm{P}=0.711)$.

\section{Discussion}

CoA has been classically defined as a narrowing of the aorta in the region of the ligamentum arteriosum (11), 
representing a spectrum of aortic narrowing from a discrete constriction of the AOi to tubular hypoplasia, with many variations between these two extremes (12). Immediate intervention after birth is required in critical cases to maintain ductal patency; therefore, an accurate prenatal diagnosis of CoA could reduce morbidity in neonates and relieve parental anxiety. At present, accurate diagnosis of CoA remains challenging because of its relatively low sensitivity and specificity. Prenatal diagnosis of CoA has been historically based on cardiovascular disproportion, but it is difficult to distinguish true CoA from a normal physiological right-sided dominance in the third trimester or ventricular discrepancy caused by intracardiac minor anomalies (such as left superior vena cava or ventricular septal defect). Except for previously reported mild cardiac defects, such as persistent left superior vena cava and ventricular septal defects, a potential relationship between RFOF and false positive diagnosis of CoA has recently been noted (6).

The exact pathogenesis of RFOF remains unclear, but it may be related to a localized developmental abnormality (13) or intrauterine myocarditis (14). RFOF is associated with several congenital malformations, including fetal hydrops and atrial arrhythmias (15). RFOF may cause increased blood flow in and dilation of the right heart, leading to congenital heart failure and fetal hydrops (12). Previous studies have also concluded that the pressure exerted by the redundant primum on the atria could cause ectopic primum in the fetus (16). The findings of our study show that the proportion of fetuses with RFOF is significantly greater in patients without CoA. In fetuses with RFOF, the pulmonary venous return will increase left atrial pressure and both the foramen flap and the atrial septum will be repositioned after birth; thus, the filling of the LV and the forward flow in the aortic arch may be increased. Therefore, RFOF is not pathological but may be self-limited. In the cases with true positive diagnosis of $\mathrm{CoA}$, the detection rate of RFOF is significantly lower. Complete or regional hypoplasia of aortic arch is present in the confirmed CoA cases, and the ventricular disproportion is by caused CoA but not RFOF. Regarding to cardiac morphological changes parameters, we also demonstrated that there was no statistical difference between fetuses with RFOF and fetuses with true fetal CoA, particularly in the inflow tracts (RV/LV ratio) and outflow tracts (AOi z-score and PA/AO ratio) of the left heart. The findings are similar to the previous study (6), who demonstrated that RFOF may simulate the signs detected on fetal echocardiography in patients with CoA, because the inflow tract of the LV may be obstructed by RFOF. The degree of asymmetry of the ventricular and great arteries is associated with excessive excursion of the septum primum into the LA.

The high false positive rate of prenatal diagnosis for CoA in our study can be attributed to two reasons. First, about two-thirds of the fetuses with RFOF showed reversed flow across the aortic arch isthmus, and this echocardiographic sign misled us to make a false positive diagnosis of CoA. However, this phenomenon may be caused by RFOF, impairing the filling of the LV and the flow in the aortic arch. Second, it was relatively difficult to distinguish normal physiological right-sided dominance in the third trimester during the study period, especially in the earlier stage of this study. In this cohort, 42 cases $(73.7 \%)$ were suspected to have CoA after 28 weeks of gestation. In our institution, routine third trimester scans were performed in each patient. Cardiovascular disproportion was considered to be a $\mathrm{PA} / \mathrm{AO}$ ratio $>1.5$ or $\mathrm{RV} / \mathrm{LV}$ ratio $>1.5$. According to our data, cardiovascular disproportion cannot be considered a useful parameter for predicting CoA. A meta-analysis published in 2017 demonstrated that a disproportion of PA/ AO ratio $>1.6$ was significantly associated with $\mathrm{CoA}$ (17). However, PA/AO ratio $>1.6$ had good sensitivity $(86.2 \%)$ but poor specificity $(51.8 \%)$ when used to predict CoA. Another study reported that incorporation of the ratio of $\mathrm{AOi} /$ duct diameters $<0.74$, isthmus diameter z-scores $<-2$, and presence of coarctation shelf and/or isthmus flow disturbance resulted in better diagnostic precision (18). The results of our study demonstrated that investigation of intracardiac structures is crucial. Although a combination of several cardiac parameters could increase the sensitivity and specificity for the prediction of CoA, RFOF may be confused with CoA because both cause similar morphological and hemodynamic variations in cardiac structures. In clinical practice, bulging of the FOF should be evaluated more critically in the four-chamber view, potentially decreasing the false positive and increasing the specificity of prenatal diagnosis of CoA. We can measure the maximum diameter of FOF and LA, and then calculate the FOF/LA ratio. If the FOF/LA ratio is above 0.65 , the diagnosis of CoA should be made with caution since that the indirect signs for CoA may be caused by RFOF.

\section{Limitation}

There are several limitations of this study that should be mentioned. First, the retrospective study design of our 
study meant that detailed data could not be obtained in all cases. Second, the false positive rate was relatively high. In our country, all the pregnant women will receive an ultrasound examination in the third trimester. However, we do not believe that this has a significant effect on the findings. Finally, the sample size of fetuses with truepositive diagnosis of CoA was relatively small.

\section{Conclusions}

The distribution of RFOF is significantly different between fetuses with and without CoA after birth. Cardiac parameters were not significantly different between fetuses with and without CoA after birth except RV diameter. For fetuses with suspected CoA, fetal echocardiography, including bulging of the FOF in the LA, should be investigated and thus may potentially decrease the false positive and increase the specificity of prenatal diagnosis of CoA.

\section{Acknowledgments}

We would give our acknowledgements to our participants, especially for their trust and comprehension.

Funding: This study was supported by research grant from the National Scientific Foundation Committee of China (No. 81501491 and No. 82171938).

\section{Footnote}

Reporting Checklist: The authors have completed the STROBE reporting checklist. Available at https://qims. amegroups.com/article/view/10.21037/qims-21-644/rc

Conflicts of Interest: All authors have completed the ICMJE uniform disclosure form (available at https://qims. amegroups.com/article/view/10.21037/qims-21-644/coif). The authors have no conflicts of interest to declare.

Ethical Statement: The authors are accountable for all aspects of the work in ensuring that questions related to the accuracy or integrity of any part of the work are appropriately investigated and resolved. The study was conducted in accordance with the Declaration of Helsinki (as revised in 2013). Written informed consent was obtained from their legal representatives (one person of their parents) because our participants were neonates or toddlers and the study was approved by the ethics committee of our institution. The study was conducted in accordance with the Declaration of Helsinki (as revised in 2013).

Open Access Statement: This is an Open Access article distributed in accordance with the Creative Commons Attribution-NonCommercial-NoDerivs 4.0 International License (CC BY-NC-ND 4.0), which permits the noncommercial replication and distribution of the article with the strict proviso that no changes or edits are made and the original work is properly cited (including links to both the formal publication through the relevant DOI and the license). See: https://creativecommons.org/licenses/by-nc-nd/4.0/.

\section{References}

1. Wilson AD, Rao PS, Aeschlimann S. Normal fetal foramen flap and transatrial Doppler velocity pattern. J Am Soc Echocardiogr 1990;3:491-4.

2. Belkin RN, Kisslo J. Atrial septal aneurysm: recognition and clinical relevance. Am Heart J 1990;120:948-57.

3. Stewart PA, Wladimiroff JW. Fetal atrial arrhythmias associated with redundancy/aneurysm of the foramen ovale. J Clin Ultrasound 1988;16:643-50.

4. Wolf WJ, Casta A, Sapire DW. Atrial septal aneurysms in infants and children. Am Heart J 1987;113:1149-53.

5. Gu X, Zhang Y, Han J, Liu X, Ge S, He Y. Isolated premature restriction or closure of foramen ovale in fetuses: Echocardiographic characteristics and outcome. Echocardiography 2018;35:1189-95.

6. Vena F, Donarini G, Scala C, Tuo G, Paladini D. Redundancy of foramen ovale flap may mimic fetal aortic coarctation. Ultrasound Obstet Gynecol 2020;56:857-63.

7. Tegnander E, Williams W, Johansen OJ, Blaas HG, EikNes SH. Prenatal detection of heart defects in a nonselected population of 30,149 fetuses--detection rates and outcome. Ultrasound Obstet Gynecol 2006;27:252-65.

8. Arya B, Bhat A, Vernon M, Conwell J, Lewin M. Utility of novel fetal echocardiographic morphometric measures of the aortic arch in the diagnosis of neonatal coarctation of the aorta. Prenat Diagn 2016;36:127-34.

9. Wu LH, Wang N, Xie HN, Du L, Peng R. Cardiovascular Z-scores in fetuses with tetralogy of Fallot. Ultrasound Obstet Gynecol 2014;44:674-81.

10. Fleiss J. Statistical methods for Rates and Proportions (2nd edn). John Wiley and Sons: New York; 1981.

11. Liu L, He YH, Li ZA, Cui CY, Zhang LZ, Li T, Liao SX, Fan TB, Peng BT, Yao HM, Huang L. Analysis of etiology, chromosome and prognosis for small left heart 
system development in 69 fetuses. J Matern Fetal Neonatal Med 2016;29:493-503.

12. Kenny D, Hijazi ZM. Coarctation of the aorta: from fetal life to adulthood. Cardiol J 2011;18:487-95.

13. Devadasan S, Batra M, Balakrishnan B, Sreeja PS, Swapneel NP. Prenatal diagnosis of isolated redundant foramen ovale: a case report. J Fetal Med 2018;5:159-62.

14. Lev M, Arcilla R, Rimoldi HJ, Licata RH, Gasul BM. Premature narrowing or closure of the foramen ovale. Am Heart J 1963;65:638-47.

15. Abu-Rustum RS, Abi-Nader KN, Zaghloul M. Prenatal diagnosis of restrictive foramen ovale: a case report. Ultrasound in Obstet Gynecol 2010; 36:168-305.

16. Papa M, Fragasso G, Camesasca C, Di Turi RP, Spagnolo

Cite this article as: Peng R, Zheng Q, He M, Shang JH, Xie HN. Comparisons of foramen ovale flap in the fetuses with true and false positive diagnosis of coarctation of the aorta. Quant Imaging Med Surg 2022;12(4):2303-2310. doi: 10.21037/ qims-21-644
D, Valsecchi L, Calori G, Margonato A. Prevalence and prognosis of atrial septal aneurysm in high risk fetuses without structural heart defects. Ital Heart J 2002;3:318-21.

17. Familiari A, Morlando M, Khalil A, Sonesson SE, Scala C, Rizzo G, Del Sordo G, Vassallo C, Elena Flacco M, Manzoli L, Lanzone A, Scambia G, Acharya G, D'Antonio F. Risk Factors for Coarctation of the Aorta on Prenatal Ultrasound: A Systematic Review and Meta-Analysis. Circulation 2017;135:772-85.

18. Jowett V, Aparicio P, Santhakumaran S, Seale A, Jicinska H, Gardiner HM. Sonographic predictors of surgery in fetal coarctation of the aorta. Ultrasound Obstet Gynecol 2012;40:47-54. 